\title{
Mineração
}

\section{Novo modelo de reconciliação para a indústria do ouro}

\section{New reconciliation model for gold industry}

\author{
Ana Carolina Chieregati \\ Dra., Professora do Departamento \\ de Engenharia de Minas e de Petróleo, \\ Escola Politécnica da USP. \\ ana.chieregati@poli.usp.br
}

\section{Luiz Eduardo Campos Pignatari}

Engenheiro de Minas, YamanaGold.

Ipignatari@yamana.com

\section{Homero Delboni Jr.}

Dr., Professor do Departamento

de Engenharia de Minas e de Petróleo,

Escola Politécnica da USP.

hdelboni@usp.br

\section{Resumo}

A reconciliação pode ser dividida em etapas, cada etapa representando o desempenho de uma das seguintes operações mineiras: estimativa de longo-prazo, estimativa de curto-prazo, planejamento, lavra e beneficiamento. $\mathrm{Na}$ indústria do ouro, ainda existe a etapa do budget ou orçamento, quando a empresa informa ao mercado sua previsão de produção anual. A divisão da reconciliação em etapas permite, não só aumentar a confiabilidade na informação do orçamento anual das empresas de mineração, mas, também, detectar e corrigir as etapas críticas responsáveis pelo erro global de estimativa, otimizando métodos e equipamentos de amostragem. O presente trabalho objetiva desenvolver e validar um novo modelo de reconciliação para a indústria do ouro. Esse modelo fundamenta-se em práticas corretas de amostragem e na subdivisão da reconciliação em etapas, visando a uma melhor estimativa de teores e a um controle mais eficiente dos processos de mineração, desde a estimativa de recursos até a produção final.

Palavras-chave: Reconciliação, amostragem, ouro.

\section{Abstract}

Reconciliation can be divided into stages, each stage representing the performance of a mining operation, such as: long-term estimation, short-term estimation, planning, mining and mineral processing. The gold industry includes another stage which is the budget, when the company informs the financial market of its annual production forecast. The division of reconciliation into stages increases the reliability of the annual budget informed by the mining companies, while also detecting and correcting the critical steps responsible for the overall estimation error by the optimization of sampling protocols and equipment. This paper develops and validates a new reconciliation model for the gold industry, which is based on correct sampling practices and the subdivision of reconciliation into stages, aiming for better grade estimates and more efficient control of the mining industry's processes, from resource estimation to final production.

Keywords: Reconciliation; sampling; gold.

\section{Introdução}

A avaliação econômica, o planejamento de lavra e a previsão de desempenho de uma operação mineira são feitos com base em estimativas de teor e de quantidade do minério. Essas estimativas são baseadas em amostras e geram os resultados de reconciliação. Quando se trata de metais preciosos, os métodos de amostragem e reconciliação são questionados, dada a dificuldade em se responder à seguinte questão: qual a representatividade da amostra e, conseqüentemente, qual a confiabilidade nos resultados de reconciliação?

A amostragem de metais preciosos é, hoje, um dos maiores desafios da 
indústria mineral, visto que, provavelmente, não existe outro tipo de material para o qual a precisão e a acurácia da amostragem sejam tão críticas. $\mathrm{O}$ ouro, em especial, tem suas peculiaridades, principalmente no que diz respeito ao efeito de segregação. A densidade do ouro é elevadíssima, promovendo uma forte segregação assim que a liberação do metal é atingida, o que dificulta a homogeneização do material. Além disto, devido ao elevado efeito pepita associado aos depósitos de ouro em geral, o teor de ouro de uma subamostra analítica pode ser completamente diferente do teor de ouro da amostra original. Esses problemas tendem a se agravar quanto menor for o teor do metal, quanto mais marginal for o depósito e quanto mais errática for a distribuição do metal no depósito, características comuns a diversos depósitos brasileiros.

Pierre Gy $(1992,1998)$ alertou os profissionais da indústria mineral sobre os problemas supracitados e afirmou que a amostragem não recebia a atenção merecida, apesar de representar a operação básica para um controle eficaz dos processos de mineração. Para a indústria do ouro, em especial, o resultado da pouca atenção dada, historicamente, à seleção de amostras corretas foi a perda anual de milhões de dólares em razão de imprecisões nas estimativas de produção. Esses prejuízos

\section{Materiais e métodos}

\section{O erro global de estimativa}

A amostragem está presente em todas as etapas de um empreendimento mineiro: desde a estimativa de recursos e reservas, perfuração, lavra, preparação da amostra e análise química até o beneficia- foram causados pela avaliação errônea do teor e da quantidade de minério existente na jazida, pela baixa previsibilidade do desempenho das operações e pelo envio de minério à pilha de estéril e de estéril à usina de beneficiamento, provocando, respectivamente, perda e diluição do minério. Todos esses problemas diminuem a confiabilidade nos resultados de reconciliação.

No contexto da indústria mineral, a reconciliação pode ser definida como uma comparação entre as estimativas dos modelos e a produção da usina. A prática comum de reconciliação baseia-se no cálculo do Mine Call Factor (MCF) e sua aplicação às estimativas dos modelos de longo e de curto-prazo, visando a melhorar a previsão do desempenho de uma operação (Morley, 2009). O MCF expressa a diferença entre a produção prevista pelos modelos e a produção registrada na usina e, portanto, sua aplicação permite uma correção das estimativas dos modelos. Esta é uma prática de "reconciliação reativa". Entretanto, a aplicação de fatores às estimativas dos modelos pode mascarar as causas dos erros responsáveis pelas discrepâncias observadas. Esses erros são devidos a métodos de modelagem inadequados, protocolos e equipamentos de amostragem incorretos, contaminação da amostra, entre outros fatores, o que prejudica a confiabilidade das estimativas.
Uma prática adequada de reconciliação deve detectar as causas dos erros entre as estimativas dos modelos e a produção observada. Eliminando-se as causas desses erros, as estimativas tornam-se prognósticos, ou seja, seus valores tendem a se aproximar do teor e da quantidade real do minério avaliado. Este é o conceito de "reconciliação pró-ativa", um processo iterativo que permite a correção das metodologias de amostragem e de estimativa de produção, visando a melhorar a previsibilidade dos modelos, sem a necessidade de aplicação de fatores às suas estimativas (Chieregati et al., 2008). A reconciliação pró-ativa é a chave de que a empresa dispõe para demonstrar que as informações divulgadas sobre recursos, reservas e desempenho de suas operações são precisas, acuradas e auditáveis (Morley \& Thompson, 2006).

Sabe-se que uma estimativa de qualidade somente é possível com práticas adequadas de amostragem, capazes de gerar amostras representativas. Conseqüentemente, a confiabilidade nos resultados de reconciliação depende da representatividade das amostras que geraram esses resultados. Conclui-se, assim, que a previsibilidade de qualquer operação mineira, desde a estimativa de recursos até a produção do metal, depende da otimização de uma operação básica, mas pouco valorizada, que é a amostragem. mento de minérios e a expedição. Apesar da importância da amostragem, pouca atenção lhe foi dada durante décadas. Felizmente, os últimos 20 anos mostraram um restabelecimento do interesse de

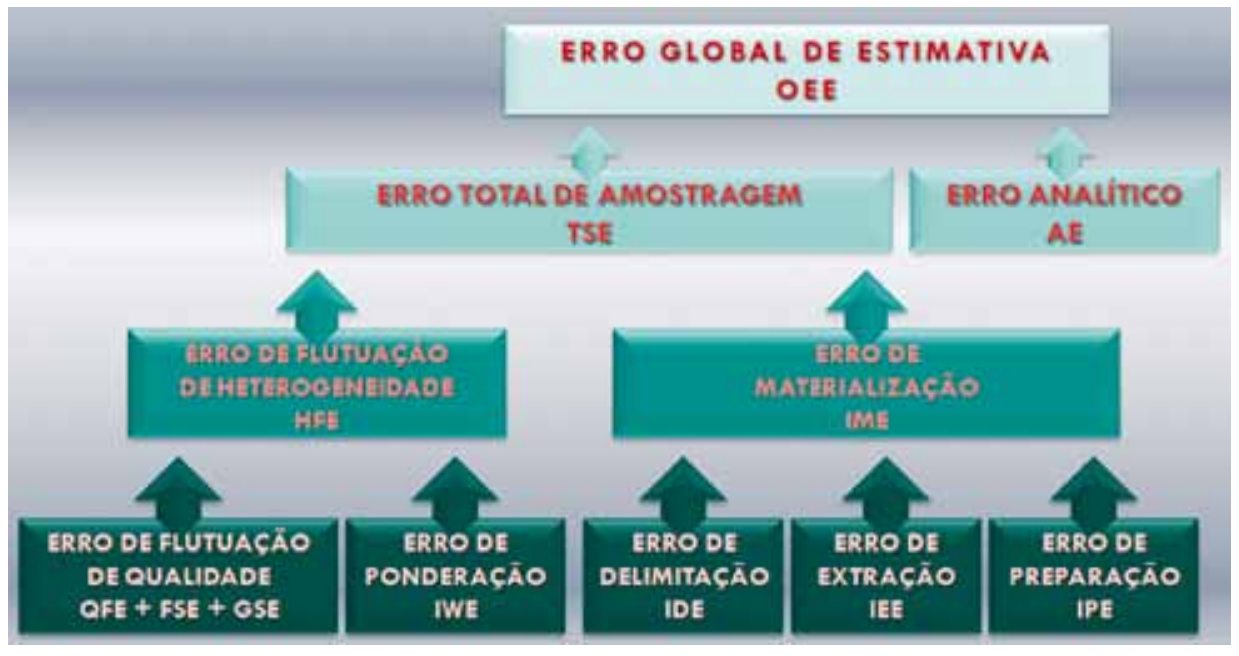

pesquisa na Teoria da Amostragem, com resultados excelentes para a indústria mineral (François-Bongarçon, 2008).

Quando uma amostra é extraída de uma massa maior de minério, ocorrem os

Figura 1

Representação

dos componentes do erro global

de estimativa (Pitard, 2010). 
erros de amostragem. Esses erros são gerados em cada etapa de seleção e de preparação da amostra e, segundo Gy (1998), um procedimento de amostragem inadequado pode levar a erros de até $1.000 \%$ para amostragem primária (seleção da amostra), de até $50 \%$ para amostragem secundária (preparação da amostra) e de 0,1 a 1,0\% para as etapas de análise química. Toda estimativa se baseia em amostras e, portanto, o erro global de estimativa inclui todos os erros de amostragem e o erro de análise química.

A Figura 1 apresenta os erros de amostragem propostos na Teoria da Amostragem de Pierre Gy. Os nomes dos erros foram traduzidos para o português, entretanto as notações foram mantidas em inglês e referem-se às notações criadas por Gy (1992) e modificadas por Pitard (1993 e 2009). O novo modelo de reconciliação proposto, nesse trabalho, está fundamentado na Teoria de Amostragem de Gy, a qual sugere procedimentos de amostragem capazes de minimizar esses erros.

\section{Relação entre amostragem e reconciliação}

Uma reconciliação bem-sucedida pode ser ilusória. Em muitos casos, erros cometidos em alguma etapa do processo são compensados por erros cometidos em outra etapa, resultando em reconciliações excelentes. Porém esse fato mascara os enviesamentos do sistema, que, mais cedo ou mais tarde, podem vir à tona (Crawford, 2004). A principal e mais óbvia fonte de erros é a amostragem. O enviesamento da amostragem é, provavelmente, o erro mais difícil de se medir e, certamente, merece uma consideração especial.

Segundo Gy (1998), “a estimativa de boa qualidade é uma corrente, e a amostragem é seu elo mais fraco.” Toda estimativa, desde a estimativa de recursos até a estimativa de produção, é baseada em amostras. Se as amostras não são representativas, as estimativas não são confiáveis. E, portanto, não tem sentido falar em reconciliação. A confiabilidade nos resultados da reconciliação depende da confiabilidade ou representatividade das amostras que geraram esses resultados.

Em se tratando de metais preciosos, os problemas de representatividade da amostra são ampliados de uma maneira

Figura 2

Os componentes de $\mathrm{v}(0)$ : "the garbage can" (Pitard, 2009).

Nota-se, assim, a importância de se conhecerem e de se quantificarem os erros considerável. Nesse caso, o efeito pepita elevado indica a baixa reprodutibilidade das amostras. Portanto esses tipos de mineralização são muito sensíveis ao método de amostragem, o qual deve ser o mais acurado possível, de modo a evitar que valores incorretos de efeito pepita sejam considerados nos modelos da jazida.

O reconhecimento do efeito pepita é de extrema importância para as estimativas dos modelos. Quanto maior o efeito pepita, maior o grau de suavização necessário na estimativa de recursos, pois a estimativa, nesse caso, aproxima-se de uma simples média aritmética das amostras contidas dentro do raio de influência do bloco em estudo (Chieregati \& Pitard, 2009). É importante ressaltar que o efeito pepita não é um fenômeno exclusivo dos metais e minerais preciosos, mas existe para qualquer material heterogêneo. Como todo material geológico é necessariamente heterogêneo, o efeito pepita ocorre para qualquer tipo de minério. O chamado "efeito pepita" da Geoestatística, ou $\mathrm{v}(0)$ do variograma, pode ser definido como a comparação entre uma unidade (ou amostra) com sua duplicata (o mais próximo dela mesma), o que, logicamente, nos levaria a concluir que $\mathrm{v}(0)$ $=0$. Na prática, conforme observou Gy (1998), não estamos lidando com massas e teores reais (sempre desconhecidos), mas com estimativas de massa e teor provenientes de processos de coleta, preparação e análise de amostras. Portanto todos os erros gerados durante esses processos estão contidos no valor v(0), chamado por Pitard (1993) de "garbage can", ou "lata de lixo", por representar a soma de todos os seguintes componentes (como mostra a Figura 2):

- Variância do erro fundamental $\left(\mathrm{s}^{2}{ }_{\mathrm{FSE}}\right)$ gerado durante a extração dos incrementos.

- Variância da heterogeneidade intrínseca do material, ou efeito pepita in situ $\left(\mathrm{s}^{2}{ }_{\mathrm{NE}}\right)$. - Variância do erro de segregação e agrupamento $\left(\mathrm{s}^{2}{ }_{\mathrm{GSE}}\right)$ também gerado durante a extração dos incrementos.

- Variância de todos os outros componentes do erro de amostragem $\left(\mathrm{s}^{2}{ }_{\mathrm{IDE}}\right.$, $\mathrm{s}_{\text {IEE }}^{2}, \mathrm{~s}_{\text {IWE }}^{2}$.

- Variância dos erros gerados durante a redução de massa das amostras até a massa enviada às análises químicas $\left(\mathrm{s}_{\mathrm{IPE}}^{2}\right)$. - Variância do erro analítico $\left(\mathrm{s}^{2}{ }_{\mathrm{AE}}\right)$.

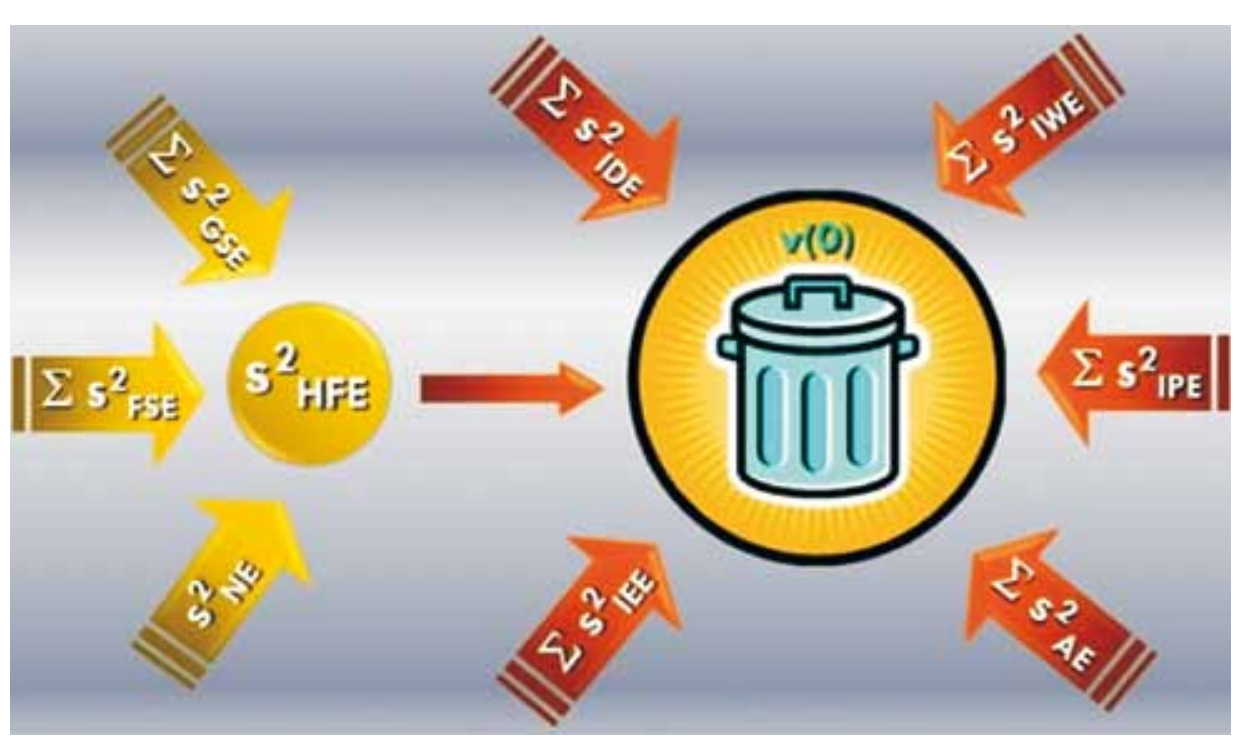

de amostragem, condição básica para a aplicação do modelo de reconciliação proposto. 
A reconciliação é uma atividade desenvolvida na maior parte das empresas de mineração e pode ser definida como a comparação entre uma medição e uma estimativa, ou seja, entre a produção e a estimativa de produção. Divide-se o teor de metal produzido pelo teor estimado pelo modelo e o resultado dessa divisão fornece o fator MCF, aplicado a futuras estimativas do modelo visando a melhorar a previsão de desempenho da operação.

Entretanto essa não é a melhor prática industrial de reconciliação. $\mathrm{O}$ principal objetivo de qualquer sistema de reconciliação não deveria ser o de gerar fatores utilizados para corrigir estimativas, mas o de permitir ajustes nos processos de modo que os resultados estejam sempre dentro de limites aceitáveis.

\section{O novo modelo de reconciliação - reconciliação pró-ativa}

A reconciliação pró-ativa é uma alternativa à reconciliação reativa e consiste em se coletarem e se analisarem dados críticos, que são usados para calibrar estimativas em um processo iterativo (Morley, 2003). Os erros são analisados com a finalidade de se tomarem ações corretivas para assegurar que a diferença entre os valores estimados e os valores reais seja mínima. Desta maneira, as estimativas tornam-se previsões - ou prognósticos - e podem formar uma base para a tomada de decisões, assegurando que o que acontecerá no futuro corresponde ao que foi planejado no presente. Portanto a reconciliação pró-ativa permite uma correção das metodologias de coleta de dados e de modelagem e não simplesmente uma correção das estimativas dos modelos aplicando-lhes fatores.

A reconciliação representa uma ferramenta poderosa para a detecção de problemas nas operações, fornecendo um diagnóstico para a resolução desses problemas. Entretanto a reconciliação não deve, simplesmente, avaliar os modelos com base nos resultados da usina (Crawford, 2004). Para que se possa visualizar as etapas críticas da operação como um todo e resolver cada problema separadamente, deve-se dividir a reconciliação em etapas.

O novo modelo de reconciliação define cinco outros fatores de reconciliação, os quais funcionam como indicadores de controle ou desempenho de cada etapa individual do empreendimento mineiro, permitindo a deteç̧ão e a correção de eventuais problemas separadamente. Em geral, esses problemas resultam de práticas inadequadas de amostragem e de modelagem da jazida, que devem, então, ser otimizadas.

A Figura 3 esquematiza o novo modelo de reconciliação proposto e seus fatores/indicadores:

- MCF ou Mine Call Factor: indica a previsibilidade do modelo de longoprazo.

- MM ou Mine Model: indica a consistência do modelo de longo-prazo.

- MP ou Mine Planning: indica a utilidade do modelo de curto-prazo para o planejamento.

- MO ou Mine Operation: indica o desempenho da operação de lavra.

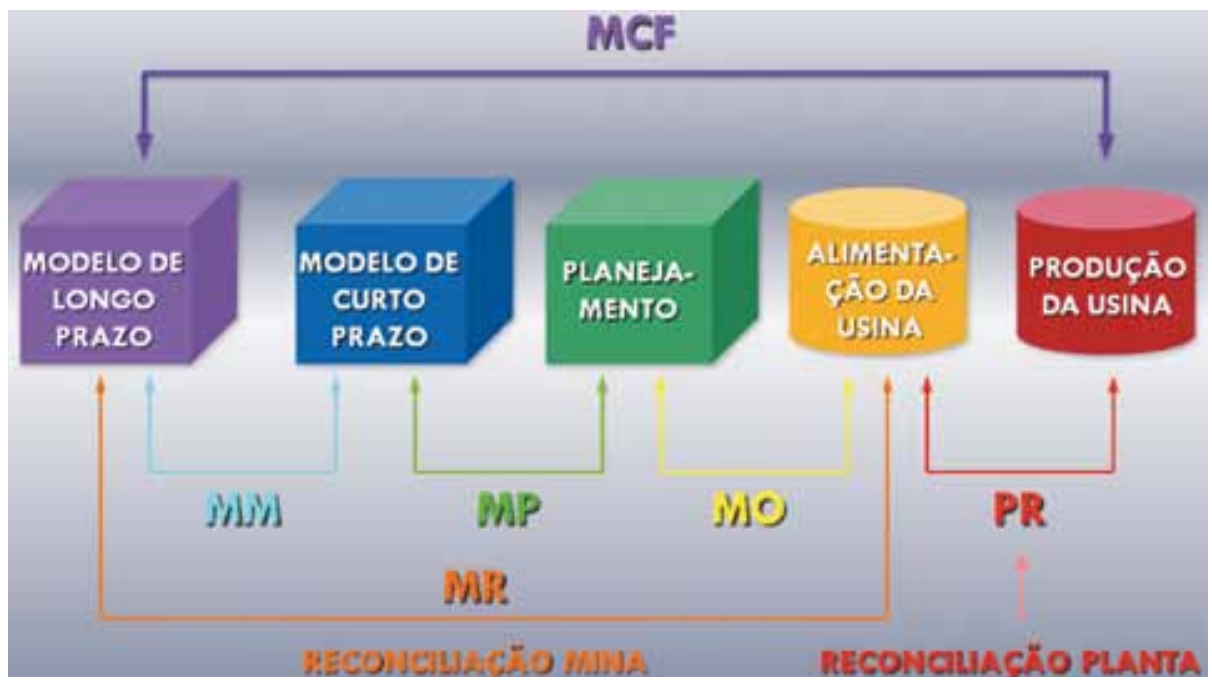

- MR ou Mine Reconciliation: indica a qualidade das estimativas do modelo de longo-prazo.

- PR ou Plant Reconciliation: indica o desempenho da operação de beneficiamento.

Cada indicador é calculado dividindo-se o valor da variável estudada (massa, teor ou conteúdo metálico) de uma etapa pelo valor da variável na etapa anterior. Por exemplo: se o modelo de curto-prazo informa que um bloco ou polígono de minério tem 2,55 g/t de $\mathrm{Au}$, baseado em amostragens adicionais segundo malha fechada, e o modelo de longo-prazo informa, para o mesmo bloco ou polígono, um valor de 2,76 g/t, então $M M=2,55 / 2,76$ $=0,92$ ou $92 \%$. Esse número indica que o modelo de longo-prazo superestima o valor do bloco, que é $8 \%$ menor segundo o modelo de curto-prazo.

No modelo proposto, os indicadores não são usados para a fatoração de estimativas, mas somente para a identificação de problemas. Vale ressaltar que o valor do MCF inclui todos os indicadores, pois é o resultado do produto entre eles, como mostra a Figura 4.

Figura 3

O novo modelo

de reconciliação proposto.

\section{Resultados}

A Figura 4 apresenta os valores dos indicadores de controle para a mina de 
denciando as etapas críticas da operação, quais sejam: (1) operação de lavra com perda de $8 \%$ do ouro planejado $(\mathrm{MO}=$ $0,92)$ e $(2)$ desempenho da usina com perda de $9 \%$ do ouro alimentado $(\mathrm{PR}=$ $0,91)$. Vale salientar que os valores dos

Figura 4

Exemplo de aplicação do novo modelo de reconciliação

(El Peñon).

\section{mostram que:}

- $\mathrm{MM}=98 \%$ : o modelo de longoprazo está consistente (a estimativa do modelo de curto-prazo para o realce estudado é apenas $2 \%$ menor que a do modelo de longo-prazo).

- $\mathrm{MP}=100 \%$ : o planejamento segue o modelo de curto-prazo.

- $\mathrm{MO}=92 \%$ : a operação de lavra fatores e indicadores não foram calculados com base no teor, mas com base no conteúdo metálico (onças de Au).

Considerando que todos os procedimentos de amostragem e preparação de amostras tenham sido realizados de maneira correta, o valor do MCF de 0,82 indica que, para o realce analisado, produziu-se $82 \%$ do ouro previsto pelo modelo de longo-prazo, nada dizendo sobre cada operação individual. Os indicadores de controle, por sua vez,

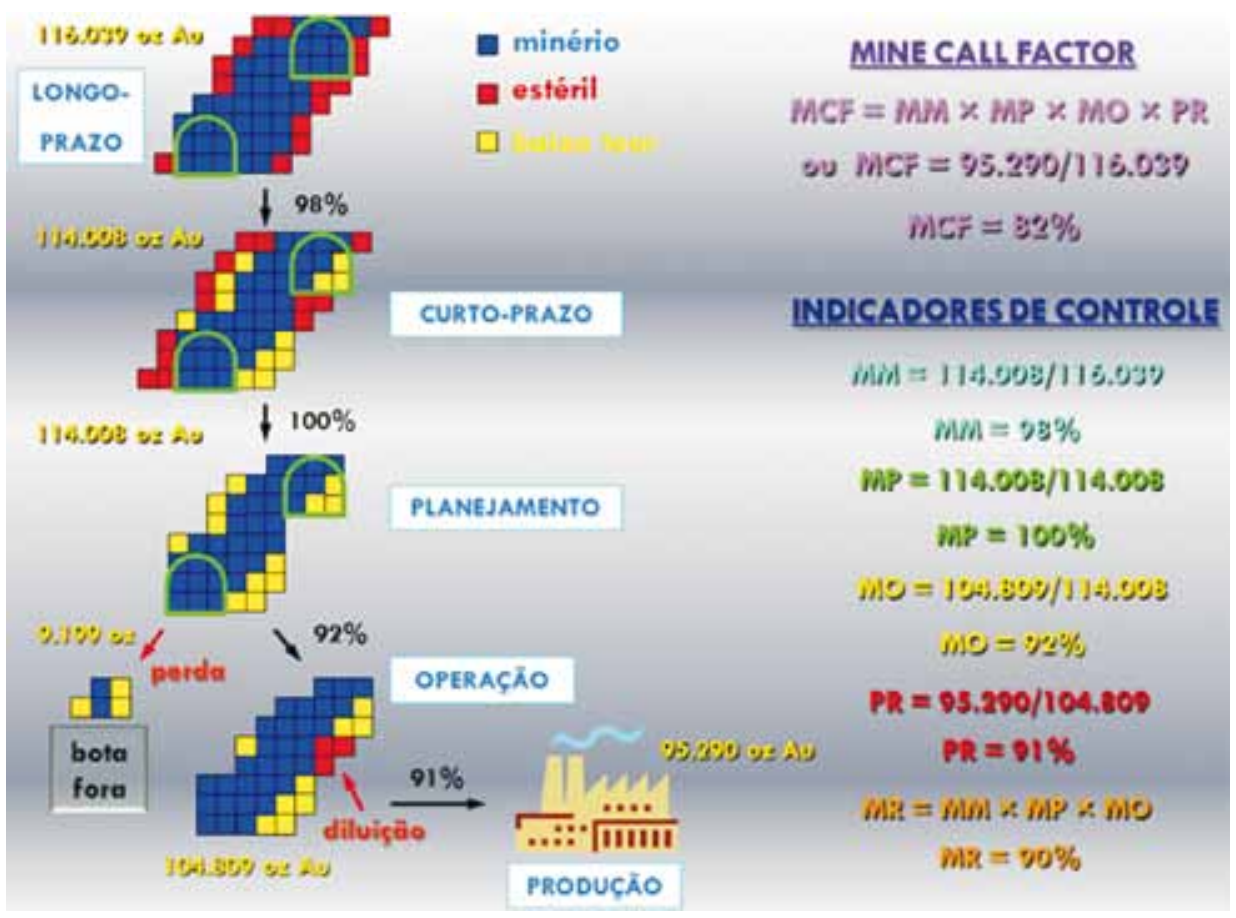

recupera $92 \%$ do ouro previsto pelo planejamento.

- $\mathrm{PR}=91 \%$ : a usina recupera $91 \%$ do ouro alimentado.

A Figura 5 apresenta os valores dos indicadores de controle para a mina de Fazenda Brasileiro (Teofilândia/BA) da YamanaGold, evidenciando as etapas críticas da operação, quais sejam: (1) modelo de longo-prazo com superestimativa de $8 \%$ do ouro reportado pelo modelo de curto-prazo (MM = 92\%); (2) operação de lavra com perda de $10 \%$ do ouro planejado $(\mathrm{MO}=0,90)$ e $(3)$ recuperação da usina com perda de $14 \%$ do ouro alimentado $(\mathrm{PR}=0,86)$.

Concomitantemente ao cálculo dos indicadores de Fazenda Brasileiro,

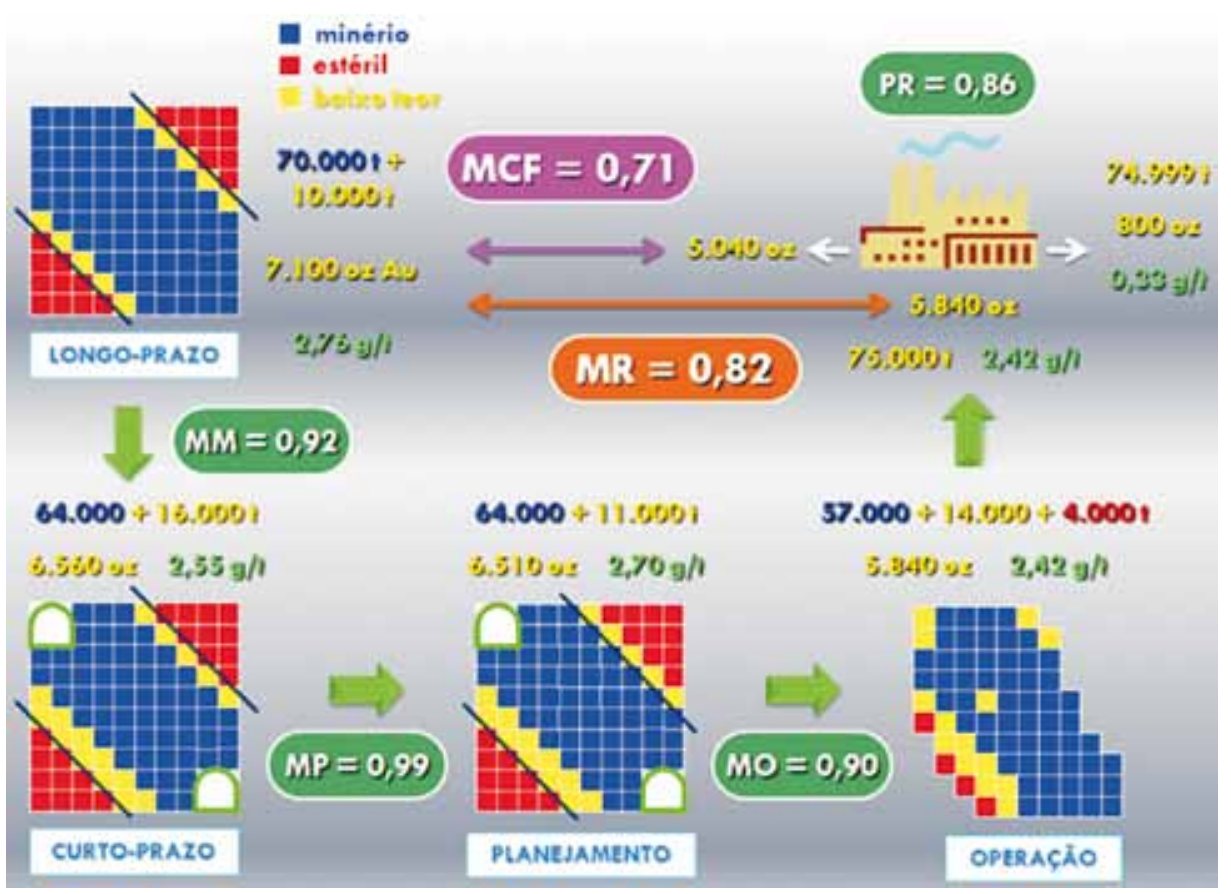


realizou-se um trabalho de otimização do procedimento de coleta de amostras na alimentação da usina, visando a aumentar a confiabilidade dos fatores $\mathrm{MO}$ e PR. O resultado desse trabalho, que calculou e analisou o erro de flutuação da heterogeneidade (HFE), mostrou a necessidade de se diminuir o intervalo de corte dos amostradores da alimentação dos moinhos, aumentando, assim, a representatividade das amostras pela minimização de HFE.

\section{Discussão}

Os resultados das duas operações estudadas mostraram que um maior enfoque deve ser dado à usina no sentido de aumentar seu desempenho, afinal, o indicador PR foi o que mais contribuiu para o MCF de ambas as operações. Antes disto, alguns trabalhos foram realizados visando a aumentar a confiabilidade das amostras e, assim, verificar a validade dos indicadores de reconciliação. Esses trabalhos incluíram o cálculo do erro de flutuação de heterogeneidade (HFE), do erro de preparação (IPE), do erro de análise química (AE) e do efeito pepita in situ do material (NE). Detalhes sobre os procedimentos para o cálculo desses erros podem ser encontrados em Pitard (1993). Tendo garantido a minimização dos erros de amostragem, procede-se ao cálculo dos indicadores para o bloco ou realce seguinte, no processo iterativo da reconciliação pró-ativa.

A análise das Figuras 4 e 5 mostra a complexidade de um sistema de reconci-

\section{Conclusão}

Atualmente, todas as empresas produtoras de ouro fomentam seus negócios com ações nos mercados de capital, pulverizando o seu controle acionário e sendo intensamente controladas e auditadas por instituições financeiras e bolsa de valores.

$O$ valor de mercado de uma empresa produtora de ouro está diretamente relacionado com suas reservas e a confiabilidade de seus planos de produção e isto só pode ser efetivamente verificado se houver um sistema adequado de reconciliação entre as reservas e a produção efetiva de conteúdo metálico da empresa. Uma reconciliação bem analisada gera possibilidades de elaborar orçamentos confiáveis ano a ano e manter um quadro de life of mine realista.

Em um passado recente, muitas das grandes minerações de ouro no liação. O cálculo dos fatores/indicadores com base no conteúdo metálico não diz nada sobre a diluição do minério ou a massa processada na usina. Pode-se concluir, então, que melhor seria calcular os fatores com base no teor e não no conteúdo metálico. Na verdade, todas essas variáveis devem ser consideradas em um sistema integrado de reconciliação, para o controle eficaz da operação.

Portanto, em se tratando de reconciliação, permanecem as seguintes questões: (1) quais indicadores usar para o controle efetivo de uma operação? (2) deve-se reconciliar massa, teor ou conteúdo metálico? (3) qual a confiabilidade de cada indicador? (4) os resultados indicam problemas de desempenho da operação ou problemas de amostragem? (5) qual a definição do MCF? Produção dividida pela estimativa do modelo de longo ou de curto-prazo? Ou seria a alimentação da usina, e não a produção, dividida pela estimativa dos modelos?

Brasil operaram com teores elevados, porém a realidade atual é muito diferente, com teores progressivamente menores em depósitos cada vez mais complexos. Quanto mais baixo o teor de ouro na jazida, maior o volume lavrado para que se possam atingir determinados índices econômicos de atratividade. Nesse ambiente, erros que causem uma maior diluição e/ou descarte de minério são determinantes do desempenho do empreendimento.

Visto que as dificuldades de se obter uma amostra representativa tornamse maiores quanto mais baixo o teor da jazida e quanto maior sua complexidade geológica, a indústria do ouro requer, mais do que nunca, o desenvolvimento de métodos de amostragem capazes de gerar amostras corretas e, portanto, estimativas acuradas e precisas da produção do metal.
Respostas a estas questões variam de operação para operação e serão consistentes somente quando práticas de reconciliação pró-ativa são usadas durante toda a vida da mina. O processo iterativo associado a essas práticas permite que mudanças nas operações e/ou procedimentos de amostragem mostrem o impacto das diversas decisões tomadas durante o processo. Os resultados das ações tomadas no passado permitem, então, realizar novas mudanças, visando a levar os indicadores o mais próximo possível de 1 , o que significa que as estimativas tornaram-se prognósticos e podem ser usadas com confiança nas tomadas de decisão e nos orçamentos anuais da empresa.

O modelo aqui proposto, aplicado à indústria do ouro, indica um caminho para o melhor controle de operações mineiras e pode ser personalizado para cada empreendimento ou tipo particular de jazida.

\section{Agradecimentos}

O presente trabalho foi realizado com o apoio do $\mathrm{CNPq}$, Conselho $\mathrm{Na}$ - cional de Desenvolvimento Científico e Tecnológico, Brasil.
A prática inovadora de reconciliação pró-ativa e a divisão da reconciliação em etapas podem auxiliar na detecção dos erros de amostragem e das etapas críticas do empreendimento, permitindo a correção e a otimização de suas operações.

Portanto o desenvolvimento do novo modelo de reconciliação proposto, nesse trabalho, pode contribuir, significativamente, para a atividade mineira de ouro no Brasil. Minerações de grande ou pequeno porte podem se beneficiar do método proposto, visto que, adotando técnicas que diminuem as oscilações de produção e aumentam o controle e a previsibilidade da operação, faria diminuir, também, sua exposição a imprevistos nos fluxos de caixa, contribuindo para a estabilidade e a sustentabilidade em um ambiente marcado por incertezas. 


\section{Referências Bibliográficas}

CHIEREGATI, A. C., DELBONI JR., H., COSTA, J. F. C. L. Sampling for proactive reconciliation practices. Mining Technology: IMM Transactions section A, v. 117, p. 136-141, 2008.

CHIEREGATI, A. C.; PITARD, F. F. The challenge of sampling gold. In: WORLD CONFERENCE ON SAMPLING AND BLENDING, 4, 2009. Cape Town. Proceedings... Johannesburg: SAIMM, 2009. p. 107-112.

CRAWFORD, G. D. Reconciliation of reserves: part 2. Pincock Perspectives, n. 50, p. 1-4, jan. 2004.

FRANÇOIS-BONGARÇON, D. A brief history of Pierre Gy's sampling theory and current trends in its acceptance in the mining world. In: SAMPLING 2008. Perth. Proceedings... Melbourne: AusIMM, 2008. p. 13-14.

GY, P. M. Sampling of heterogeneous and dynamic material systems: theories of heterogeneity, sampling and homogenizing. Amsterdam: Elsevier, $1992.653 \mathrm{p}$.

GY, P. M. Sampling for analytical purposes. Chichester: John Wiley \& Sons, 1998. 153 p.

MORLEY, C. Beyond reconciliation: a proactive approach to using mining data. In: LARGE OPEN PIT MINING CONFERENCE, 5, 2003. Kalgoorlie. Proceedings... Melbourne: AusIMM, 2003. p. 185-192.

MORLEY, C. Standardising the reconciliation factors required in governance reporting. Snowden Newsletter, n. 57, p. 1-2, nov. 2009.

MORLEY, C., THOMPSON, K. Extreme reconciliation - a case study from Diavik diamond mine, Canada. In: INTERNATIONAL MINING GEOLOGY CONFERENCE, 6, 2006. Darwin. Proceedings... Melbourne: AusIMM, 2006. p. 313-321.

PITARD, F. F. Pierre Gy's sampling theory and sampling practice: heterogeneity, sampling correctness, and statistical process control. 2 ed. Boca Raton: CRC Press, 1993. 488p.

PITARD, F. F. Poisson processes in sampling. In: WORLD CONFERENCE ON SAMPLING AND BLENDING, 4, 2009. Cape Town. Workshop - Poisson Processes in Sampling... Johannesburg: SAIMM, 2009. Course notes.

PITARD, F. F. Minimization of sampling bias (elimination of incorrect sampling errors). In: HORIZONTAL STANDARD NOTES. Broomfield, 2010. (Personal notes).

Artigo recebido em 12 de setembro de 2010. Aprovado em 20 de dezembro de 2010. 